\title{
The early career, gender, and diversity actions within the LHCb collaboration
}

\author{
Barbara Sascia on behalf of the LHCb Collaboration * \\ Laboratori Nazionali dell'INFN di Frascati, Italy \\ E-mail: Barbara.Sciascia@cern.ch
}

\author{
Jonas Rademacker on behalf of the LHCb collaboration* \\ H.H. Wills Physics Laboratory, University of Bristol, UK \\ E-mail: Jonas.Rademacker@cern.ch
}

\begin{abstract}
The LHCb collaboration has, as the first (and so far only) LHC collaboration, created a dedicated office for Early Career, Gender and Diversity (ECGD). The ECGD office's role is to advise the management on ECGD matters; provide a point of contact for anybody experiencing any kind discrimination, bullying or harassment; collate regular statistics and other relevant information related to gender and, where appropriate, other ECGD matters; organise regular open meetings where ECGD matters are discussed. We report on the first two years of the LHCb ECGD office.
\end{abstract}

38th International Conference on High Energy Physics 3-10 August 2016

Chicago, USA

${ }^{*}$ Speaker (joint presentation by both authors) 


\section{Creation of the LHCb ECGD Office}

The ECDG office [2] came to existence in 2014 in response to the recommendations of the Equality and Diversity Taskforce set up by the LHCb management, and has been endorsed by the LHCb Collaboration Board. This process was triggered by study of the gender distribution in the LHCb collaboration (discussed in section 2), which was followed a collaboration-wide survey. The subsequent survey focused on gender and career issues. It showed that many early career physicists, male and female, struggle with career planning and career progression, work-life balance, and combining career and family. It also revealed that female collaboration members had experienced discrimination and sexual harassment. Many studies, for example a large-scale survey carried out by the European Union Agency for Fundamental Rights [1], show that about one third of women above the age of fifteen have experienced some form of sexual harassment, half of which at work. These studies show also that, contrary to widespread wishful thinking amongst academics, there is no indication that working in a particularly skilled or educated environment improves these numbers. That $\mathrm{LHCb}$ and its institutes are not so different from the rest of society was contrary to most collaboration members' expectation, and of course it is still contrary to the collaboration's ambitions.

The management appointed a Equality and Diversity Taskforce, and implemented its recommendations. Amongst the measures adopted is the appointment of two Early Career, Gender and Diversity (ECGD) Officers, one man and one woman, with the following mandate:

- to advise the management on ECGD matters;

- to be available for listening to and advising colleagues, in a confidential manner, who feel that they are the victims of harassment, discrimination, or other inappropriate behaviour

- to collate regular statistics and other relevant information related to gender and, where appropriate, other ECGD matters, so that the collaboration's progress in this area can be monitored

- to assist the management in scheduling regular open meetings where ECGD matters can be discussed.

The ECGD office was created in 2014, with the endorsement of the LHCb Collaboration Board, and with the authors of this contribution as its officers. Since 2015, ECGD officers are invited to attend the Collaboration Board meetings. The ECGD office does not act in isolation. Many men and women at $\mathrm{LHCb}$ have spent considerable time and effort engaging with ECGD office activities.

\section{Current gender composition of $\mathrm{LHCb}$}

For practical reasons (we have the data), this section is about gender statistics; this should not distract from the fact that the full mandate of the ECGD office covers diversity and issues of harassment and discrimination in the broadest sense. Currently, $17 \%$ of physicists at $\mathrm{LHCb}$ are female (same as in ATLAS [5]). The fraction of female physicists as a function of age is shown in figure 1. It shows that the under-representation of women increases with age, and thus, one may conjecture, with seniority. On the other hand, the increasing fraction of women amongst the 
2006

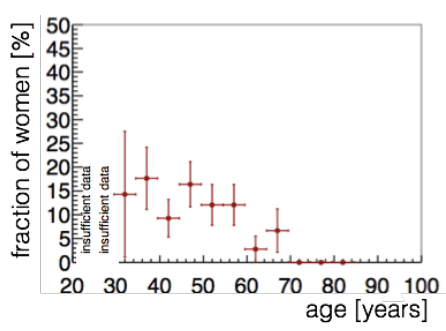

2011

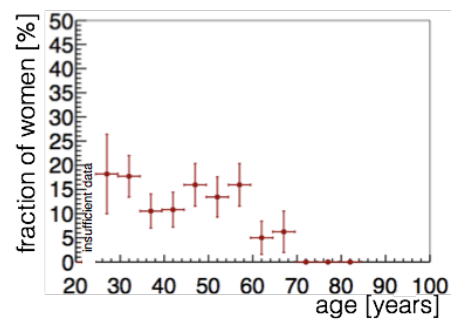

2016

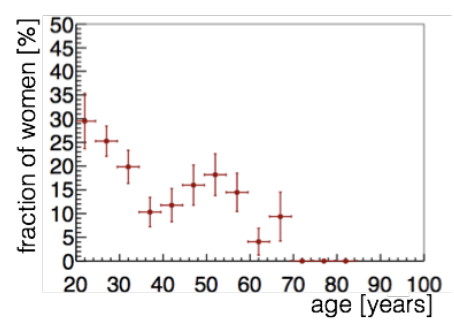

Figure 1: Fraction of women amongst LHCb physicists of different age groups in 2006, 2011, 2016. Entries where the total number of physicists (male + female) is less than 5 are excluded.

\begin{tabular}{l|ccc} 
Position & F & M & F[\%] \\
\hline Spokesperson & 0 & 4 & 0 \\
Deputy SP & 2 & 4 & 33 \\
Collaboration Board Chair & 2 & 4 & 30 \\
Technical Coordinator & 0 & 3 & 0 \\
Physics Coordinator & 0 & 6 & 0 \\
Group Leaders (2016) & 5 & 55 & 8.3 \\
Project Leaders (2016) & 2 & 12 & 16 \\
Working Group Conveners (2016) & 8 & 23 & 26
\end{tabular}

Table 1: Leadership positions in LHCb since its foundation (last three rows 2016 only)

youngest collaboration members is encouraging. But even if this increase in the female fraction of new collaboration members is sustained, its long term effects will depend on whether these young female physicists leave the field at a higher rate then their male colleagues (the "leaky pipe" effect), or not.

Table 1 shows the fraction of women in various leadership positions in the collaboration. The group leader statistics reflects that women are, as conjectured above, under-represented in senior positions, even relative to the low $17 \%$ baseline in the collaboration. Other positions of responsibility show (within large statistical errors), a picture broadly compatible with this $17 \%$ baseline. But it is very much worth questioning what the correct baseline is. The fraction of women in the collaboration (17\%), at the appropriate career stage for the position $(<17 \%$ for CB chair, $>17 \%$ for WG conveners), in the world (50\%)? How should we take into account those that left in frustration because of discrimination and discouragement, or never even entered the field for those reasons? Either way, there is widespread consensus in the collaboration that we should remove obstacles for women and for minority groups to enter the field, progress their careers, and enter leadership positions. To help the collaboration achieve this is part of the ECGD mandate.

\section{Diversity and gender-equality actions by LHCb}

Reinstatement after (m/p)aternity leave This is a measure taken already before the ECGD office came into existence. People in positions of responsibilities like Working Group Conveners, who take long-term leave due to caring responsibilities get re-instated into these positions on re- 
turn. The effectiveness of this policy is also limited by the fact that many of those who are meant to benefit from it work on short-term contracts that are over before they can return to their job (which has of course consequences far more severe than just missing out on a convenership).

Gender balance and diversity in leadership positions Every round of appointments to working group conveners and sub-conveners is discussed with the ECGD office. The ECGD office does not interfere with the choice of convener/subconvener, but we do sometimes give advice on the process, e.g. how to handle cases where a promising candidate is about to go on maternity leave.

Speaker Appointments The speakers' bureau, together with the ECGD office, try to ensure that potential speakers do not lose out on conference talks because they do not wish to travel to certain countries, for example those with particularly homophobic legislation or widespread homophobic violence. An important measure in this respect is that refusing a talk will not count against getting one in future (no reason needs to be given); instead the speakers' bureau will actively seek to find an alternative opportunity.

Advising Individuals An important part of the ECGD's mandate is to advice individuals who have been subject to discrimination, bullying, harassment and other problems.

Welcome Letter \& Constitution In December 2016, the LHCb collaboration board ratified an amendment to its constitution, which includes the lines:

Members of the LHCb collaboration are expected to act in an ethical and collaborative manner at all times, and abide by the CERN code of conduct. LHCb members shall not engage in harassment or bullying, or discriminate against each other in particular on the basis of sex, age, religion, beliefs, nationality, culture, ethnicity, sexual orientation, gender identity, career status, disability, or family situation.

The letter welcoming new collaborators to $\mathrm{LHCb}$ also emphasises the importance the collaboration places on these issues.

ECGD Meetings The ECGD office organises plenary meetings in each LHCb Collaboration weeks, typically attended by 100-200 members (out of about 700 LHCb authors). Topics discussed were:

- Mentoring

- $(\mathrm{P} / \mathrm{M})$ aternity Leave and other career breaks

- Sexual Harassment

- HEP-What Else! HEP-What Else? Career structure in particle physics, the lack of long-term career prospects in the field, career opportunities outside particle physics.

- Representation of early career physicist in the management structure

- Gender representation 
Some of these meetings led to concrete action. We implemented a mentoring system at LHCb, and provided input to CERN's 5-yearly review on employment conditions. The meeting on careers outside physics included speakers who were LHCb members and now pursue careers outside physics - and the opportunity to network with them. We are currently building a LinkedIn network to improve the connection between current $\mathrm{LHCb}$ physicists and those who left the field, expecting that this will provide concrete help in finding jobs and learning about possible career paths. The ALICE/ATLAS/CMS/LHCb careers and networking events (LHC-wide events that took place in 2015 and 2016, which followed an initiative by CMS) pursue similar aims on a larger scale, and we are actively involved with those. The representation of early career physicists in our collaboration is now an issue discussed on many levels, and while it has not yet resulted in any concrete measures, we are confident it will.

\section{Career progression}

A large fraction of $\mathrm{PhD}$ students enter the field with the intention of pursuing an academic career $[4,3]$. The vast majority of them will leave the field. There are simply too few positions. The good news is, however, that particle physics PhDs are highly employable in the private sector [4, 3].

Obtaining one of those rare academic positions requires skill, but also luck. "If you want it hard enough, and work hard enough, you will succeed in academia" is the self-affirming mythology of the lucky ones who prefer to see their career as inevitable consequence of their own hard work and brilliance. Apart from ignoring reality, it declares the most likely outcome of someone's HEP career a failure. It also ignores the fact that there are highly stimulating and intellectually demanding careers outside HEP. This myth is particularly powerful, and damaging, because of the strong selection bias our $\mathrm{PhD}$ students are subjected to - they talk predominantly to those who had a career in academia, and not to the majority of scientists, whose career is elsewhere.

Many particle physics PhDs find themselves ill-prepared for hunting jobs in the private sector. Those who stay in academia are often worse off. They work anti-social hours; frequent changes of employer and country of residence make stable relationships, or planning a family, difficult or impossible; after a string of short-term contracts, they often find themselves still in insecure employment, too old to have a realistic prospect of an academic position, and at that point, finding it very difficult to change field. HEP training and employment needs to change.

- Planning security - scientists need to know early on in their career whether they are likely to get an academic position or not, not when it is too late to plan an alternative career. A step in the right direction is a recent initiative in Germany (see https://www.bmbf.de/de/wissenschaftlichernachwuchs-144.html), where 1000 postdoctoral position that lead by default to a professorship are being created over the next 15 years. Some universities (including Manchester, Bristol) have started fellowship programs with a similar structure.

- While the training as a scientists is the key qualification that must not be eroded, skills and qualifications that prepare $\mathrm{PhD}$ students and postdoctoral researchers for work in industry should form an intrinsic part of their training; this includes technical skills such as modern programming languages and computing platforms, as well as 'soft skills' like time management, CV writing and interview practice. It should be a normal part of a PhD student's / 
postdoctoral researcher's life to be exposed to job fares / career events like those organised by ALICE/ATLAS/CMS/LHCb.

- The macho culture in which people boast with 70 hour weeks, not taking holidays or weekends off and neglecting their friends and family, must end. A more healthy work culture in HEP is urgently needed for all our well being, and for the productivity of the field.

- Collaborations should have mechanisms in place that ensure the interests of early career scientists are taken into account in decision making processes.

Measures to help early career scientists at LHCb include: the LHCb mentoring system; helping build contacts between ex-collaboration members that left the field and current LHCb members, presentations and Q\&A sessions with former LHCb members now working in the private sector in ECGD meetings and dedicated careers meetings; an LHCb LinkedIn group; prizes for contributions to LHCb (especially including operational contributions).

\section{Conclusion and prospect}

In 2016, LHCb repeated the survey, expanding its scope beyond its initial focus on gender. The data are still being analysed. Key results include that discrimination is being experienced on a variety of grounds, including gender, race and religion; sexual harassment has not gone away; bullying at work is a key issue that needs to be addressed; support in career planning is in high demand. The survey also asked LHCb members how they felt about the activities of the ECGD office. The feedback was overwhelmingly positive. We believe that the only way in which such an office can have any significant impact is by acting from within the collaboration, rather than preaching high morals from the sidelines. While we have not changed the world, we believe that in this respect the LHCb ECGD office was a success. It is seen as an intrinsic and welcome part of the collaboration, which supports its aims and activities. We are proud of the trust the collaboration vested in us, and grateful for the support we received. In Oct 2016, a new pair of ECGD officers have taken over. They have a lot to do, but they have an excellent, supportive environment to work in.

\section{References}

[1] FRA, European Union Agency for Fundamenta Rights. Violence against women: an EU-wide survey. Main results. 2014. Most relevant in this context is section 6.

[2] LHCb ECGD Office. Webpage. http://hcb.web.cern.ch/lhcb/ECGD_Office/ECGD-intro.html.

[3] J. Pold and P. Mulvey. Physics doctorates initial employment data from the degree recipient follow-up survey for the classes of 2013 and 2014. focus on - a publication of the AIP statistical research centre, (301.209.3070), March 2016.

[4] Science and Technology Facilities Council. A study of the career paths of PPARC/STFC funded PhD students. Technical report, March 2010.

[5] The ATLAS collaboration. Studies related to gender and geographic diversity in the ATLAS Collaboration. Technical Report ATL-GEN-PUB-2016-001, CERN, Geneva, Jul 2016. 